\title{
REMARKS ON THE ASYMPTOTIC BEHAVIOR OF SOLUTIONS TO DAMPED EVOLUTION EQUATIONS IN HILBERT SPACE
}

\author{
FREDERICK BLOOM ${ }^{1}$
}

\begin{abstract}
Lower bounds are derived for the norms of solutions to a class of intitial-value problems associated with the damped evolution equation $u_{t}+$ $A u_{t}+B u=0$ in Hilbert space. Under appropriate assumptions on the linear operator $B$ it is shown that even in the special strongly damped case where $A=\Gamma I, \Gamma>0$, solutions are bounded away from zero as $t \rightarrow+\infty$, even when $\Gamma \rightarrow+\infty$.
\end{abstract}

Various authors (and most notably Dafermos [1] and Russell [2]) recently studied the asymptotic behavior of solutions to initial-value problems associated with damped linear evolution equations of the form ${ }^{2}$

$$
u_{t t}+A u_{t}+B u=0
$$

where $u:[0, \infty) \rightarrow H$, a real Hilbert space with inner-product $\langle$,$\rangle and$ natural norm $\|(\cdot)\|$; the usual assumptions which are made relative to (1) are that [2] $B$ is a bounded linear operator on $H$ which satisfies a coerciveness condition of the form

$$
\langle v, B v\rangle \geqslant \lambda\|v\|^{2} ; \quad \lambda>0, v \in \mathscr{D}(B),
$$

where $\mathscr{D}(B)$ denotes the domain of $B$ and is such that $\overline{\mathscr{D}(B)}=H$. If $\langle A v, v\rangle_{H} \geqslant 0$ and $A^{-1}$ exists (the strongly damped case) then it is well known that the energy

$$
\mathcal{E}(t)=\frac{1}{2}\left(\left\|u_{t}\right\|^{2}+\langle u(t), A u(t)\rangle\right)
$$

associated with (1) decays at a uniform exponential rate and the asymptotic stability of solutions to (1) follows immediately. Even if $A^{-1}$ does not exist (the weakly damped case) it may still be shown that under various circumstances $\lim _{t \rightarrow \infty} \delta(t)=0$. Our concern in this note will be with a special case of the strongly damped situation where $A=\Gamma I, \Gamma>0$ a real number, but where the coerciveness condition (2) is not satisfied. We consider, in fact, the system

$$
\begin{gathered}
u_{t t}^{\alpha}+\Gamma u_{t}^{\alpha}-N u^{\alpha}=0, \\
u^{\alpha}(0)=\alpha u_{0}, u_{t}^{\alpha}(0)=v_{0} \quad\left(u_{0}, v_{0} \in \mathscr{D}(N)\right)
\end{gathered}
$$

Received by the editors January 16, 1978 and, in revised form, September 4, 1978.

AMS (MOS) subject classifications (1970). Primary 47A50; Secondary 35B40.

${ }^{1}$ Research supported in part by AFOSR Grant 77-3396.

${ }^{2}(\cdot)$, denotes differentiation with respect to the time variable $t$. 
where $\alpha>0$ is a real number and $u^{\alpha} \in \mathcal{C}^{2}([0, \infty) ; H)$ such that $u^{\alpha}(t) \in$ $\mathscr{D}(N)$ for each $\alpha>0, t \in[0, \infty)$. We have replaced the operator $B$ in (1) by $-N$ in (4) so as to afford easy comparison with recent related work on growth estimates for solutions of undamped $(\Gamma=0)$ linear differential equations in Hilbert space [3], [4] which employ differential inequality arguments of the type we will use in this paper.

In the present situation the positivity assumption (2) would assume the form

$$
\langle v, N v\rangle \leqslant-\lambda\|v\|^{2}, \quad \lambda>0, v \in \mathscr{D}(N) .
$$

However, the assumptions that we will make here are that $N$ is symmetric and that $^{3}$

$$
\langle v, N v\rangle \geqslant 0, \quad v \in H
$$

with there being (at least) one element $\bar{u}_{0} \in \mathscr{D}(N)$ such that

$$
\left\langle\bar{u}_{0}, N \bar{u}_{0}\right\rangle>0
$$

under the assumptions (7a) and (7b) it will be shown that solutions $u^{\alpha}$ of (4), (5) with $u_{0}=\bar{u}_{0}$ and $\alpha$ sufficiently large satisfy

$$
\lim _{t \rightarrow \infty}\left\|u^{\alpha}(t)\right\|^{2} \geqslant \alpha^{2}\left\|\bar{u}_{0}\right\|^{2} e^{-\Sigma_{0}(\alpha, \Gamma)}
$$

where $\Sigma_{0}(\alpha, \Gamma)$ depends on $\bar{u}_{0}, v_{0}$ and satisfies $\lim _{\Gamma \rightarrow \infty} \Sigma_{0}(\alpha, \Gamma)=0$, for all $\alpha>0$, so that

$$
\lim _{\Gamma \rightarrow \infty} \lim _{t \rightarrow \infty}\left\|u^{\alpha}(t)\right\|^{2} \geqslant \alpha^{2}\left\|\bar{u}_{0}\right\|^{2}
$$

for all $\alpha$ sufficiently large.

REMARK. In [1] a second real Hilbert space $V$ is introduced with $\bar{V}=H$, $V \subset H$ algebraically and topologically. Letting $V^{\prime}$ denote the dual of $V$ via $\langle$, $\rangle$, i.e.,

$$
\|v\|_{V^{\prime}}=\sup _{w \in V} \frac{|\langle v, w\rangle|}{\|w\|_{V}}
$$

the assumption in [1] which corresponds to (2) is that

$$
\langle v, B v\rangle \geqslant \lambda\|v\|_{V}^{2}, \quad \lambda>0, v \in V,
$$

where $B \in \mathcal{L}\left(V, V^{\prime}\right)$; such a setting is particularly appropriate for dealing with problems in linear partial differential equations and it will be obvious that our results carry over immediately in this framework also.

We now state and prove the basic growth estimate for the system (4), (5) from which the claimed asymptotic results (8) and (9) follow almost immediately; the argument presented below is a hybrid mixture of logarithmic concavity and logarithmic convexity arguments of the kind which have been used so successfully in recent years to establish results on uniqueness,

${ }^{3}$ Thus (4) is an abstract version of an elliptic partial differential equation. 
stability, continuous dependence, and instability of solutions to initial-value problems for abstract linear and nonlinear equations in Hilbert space [4]-[6], as well as various initial-boundary value problems for nonlinear partial differential equations [7] $[$ [10].

THEOREM. If $u^{\alpha} \in C^{2}([0, \infty)$; $\mathscr{D}(N))$ is a solution of (4), (5), with $u_{0}=\bar{u}_{0}$, and (7a), (7b) obtain, then

$$
\left\|u^{\alpha}(t)\right\|^{2} \geqslant \alpha^{2}\left\|\bar{u}_{0}\right\|^{2} \exp \left[\left\{\frac{\left\langle\bar{u}_{0}, v_{0}\right\rangle}{\alpha \Gamma\left\|\bar{u}_{0}\right\|^{2}}\right\}\left(1-e^{-\Gamma t}\right)\right]
$$

for all $\alpha \geqslant\left\|v_{0}\right\| / \sqrt{\left\langle\bar{u}_{0}, N \bar{u}_{0}\right\rangle}$ and $t \geqslant 0$.

Proof. Let $F_{\alpha}(t)=\left\|u^{\alpha}(t)\right\|^{2}, 0 \leqslant \mathrm{t}<\infty$, where $u^{\alpha} \in C^{2}([0, \infty) ; H)$ is any solution of (4), (5) with $u_{0}=\bar{u}_{0}$ and let $\beta>0$ arbitrary. A direct computation, analogous to Levine [9, II, Theorem I], yields $\left(F_{\alpha}^{\prime} \equiv(d / d t) F_{\alpha}\right)$ :

$$
\begin{aligned}
F_{\alpha} F_{\alpha}^{\prime \prime}-(\beta & +1) F_{\alpha}^{\prime 2} \\
& =4(\beta+1) S_{\alpha}^{2}+2 F_{\alpha}\left\{\left\langle u^{\alpha}, u_{t t}^{\alpha}\right\rangle-(2 \beta+1)\left\langle u_{t}^{\alpha}, u_{t}^{\alpha}\right\rangle\right.
\end{aligned}
$$

where

$$
S_{\alpha}^{2}=\left(\left\langle u^{\alpha}, u^{\alpha}\right\rangle\right)\left(\left\langle u_{t}^{\alpha}, u_{t}^{\alpha}\right\rangle\right)-\left\langle u^{\alpha}, u_{t}^{\alpha}\right\rangle^{2} \geqslant 0,
$$

by the Schwarz inequality, for all $\alpha>0$; the result in (13) depends only on the form of $F_{\alpha}(t)$ and is independent of the particular equation satisfied by $u_{\alpha}$. In view of (4) and (14) we may deduce from (13) the differential inequality

$$
F_{\alpha} F_{\alpha}^{\prime \prime}-(\beta+1) F_{\alpha}^{\prime 2} \geqslant 2 F_{\alpha} G_{\alpha, \beta}, \quad 0 \leqslant t<\infty,
$$

where, for arbitrary $\beta>0$,

$$
G_{\alpha, \beta} \equiv\left\langle u^{\alpha}, N u^{\alpha}\right\rangle-\Gamma\left\langle u_{t}^{\alpha}, u^{\alpha}\right\rangle-(2 \beta+1)\left\langle u_{t}^{\alpha}, u_{t}^{\alpha}\right\rangle .
$$

Therefore,

$$
\begin{aligned}
G_{\alpha, \beta}^{\prime}= & -4 \beta\left\langle u_{t}^{\alpha}, N u^{\alpha}\right\rangle-\Gamma\left\langle u_{t}^{\alpha}, u_{t}^{\alpha}\right\rangle \\
& -\Gamma\left\langle u^{\alpha}, u_{t t}^{\alpha}\right\rangle+2 \Gamma(2 \beta+1)\left\|u_{t}^{\alpha}\right\|^{2}
\end{aligned}
$$

but

$$
G_{\alpha, \beta}(0)=\alpha^{2}\left\langle\bar{u}_{0}, N \bar{u}_{0}\right\rangle-\alpha \Gamma\left\langle\bar{u}_{0}, v_{0}\right\rangle-(2 \beta+1)\left\|v_{0}\right\|^{2}
$$

so integration of (17) and substitution from (18) yields

$$
\begin{aligned}
G_{\alpha, \beta}(t)= & G_{\alpha, \beta}(0)-2 \beta\left[\left\langle u^{\alpha}, N u^{\alpha}\right\rangle-\alpha^{2}\left\langle\bar{u}_{0}, N \bar{u}_{0}\right\rangle\right] \\
& -\Gamma\left[\left\langle u^{\alpha}, u_{t}^{\alpha}\right\rangle-\alpha\left\langle\bar{u}_{0}, v_{0}\right\rangle\right]+2 \Gamma(2 \beta+1) \int_{0}^{t}\left\|u_{\tau}^{\alpha}\right\|^{2} d \tau \\
\geqslant & (2 \beta+1)\left[\alpha^{2}\left\langle\bar{u}_{0}, N \bar{u}_{0}\right\rangle-\left\|v_{0}\right\|^{2}\right]-2 \beta\left\langle u^{\alpha}, N u^{\alpha}\right\rangle-\Gamma\left\langle u^{\alpha}, u_{t}^{\alpha}\right\rangle
\end{aligned}
$$

where we have dropped the term proportional to $\int_{0}^{t}\left\|u_{\tau}^{\alpha}\right\|^{2} d \tau$. However, in view (7) and the definition of $F_{\alpha}$, if we now choose $\alpha \geqslant\left\|v_{0}\right\| / \sqrt{\left\langle\bar{u}_{0}, N \bar{u}_{0}\right\rangle}$ 
then the inequality in (19) may be replaced by

$$
G_{\alpha, \beta}(t) \geqslant-2 \beta\left\langle u^{\alpha}, N u^{\alpha}\right\rangle-\frac{\Gamma}{2} F_{\alpha}^{\prime}(t) .
$$

But, by (16),

$$
\left\langle u^{\alpha}, N u^{\alpha}\right\rangle=G_{\alpha, \beta}+\Gamma\left\langle u^{\alpha}, u_{t}^{\alpha}\right\rangle+(2 \beta+1)\left\|u_{t}^{\alpha}\right\|^{2}
$$

and so substituting in (20) from (21) and solving for $G_{\alpha, \beta}$ we find that

$$
G_{\alpha, \beta} \geqslant-\Gamma \mu(\beta) F_{\alpha}^{\prime}-2 \beta\left\|u_{t}^{\alpha}\right\|^{2}
$$

where

$$
\mu(\beta) \equiv\left(\beta+\frac{1}{2}\right) /(2 \beta+1) .
$$

Now, suppose we take the inner-product of (4) with $u^{\alpha}$, i.e.,

$$
\left\langle u^{\alpha}, u_{t i}^{\alpha}\right\rangle+\Gamma\left\langle u^{\alpha}, u_{t}^{\alpha}\right\rangle-\left\langle u^{\alpha}, N u^{\alpha}\right\rangle=0 .
$$

Then it is easily seen that this equation may be rewritten in the form

$$
\frac{1}{2} \frac{d^{2}}{d t^{2}}\left\|u^{\alpha}\right\|^{2}+\frac{\Gamma}{2} \frac{d}{d t}\left\|u^{\alpha}\right\|^{2}=\left\|u_{t}^{\alpha}\right\|^{2}+\left\langle u^{\alpha}, N u^{\alpha}\right\rangle
$$

which yields, in view of (7a) and the definition of $F_{\alpha}$, the inequality

$$
\left\|u_{t}^{\alpha}\right\|^{2}<\frac{1}{2} F_{\alpha}^{\prime \prime}+\frac{\Gamma}{2} F_{\alpha}^{\prime}
$$

Combining (22) and (25) we have

$$
G_{\alpha, \beta} \geqslant-\Gamma(\beta+\mu(\beta)) F_{\alpha}^{\prime}-\beta F_{\alpha}^{\prime \prime}
$$

and, therefore, in view of (15),

$$
F_{\alpha} F_{\alpha}^{\prime \prime}-(\beta+1) F_{\alpha}^{\prime 2} \geqslant-2 \Gamma(\beta+\mu(\beta)) F_{\alpha}^{\prime} F_{\alpha}-2 \beta F_{\alpha} F_{\alpha}^{\prime \prime}
$$

or

$$
F_{\alpha} F_{\alpha}^{\prime \prime}-[(\beta+1) /(2 \beta+1)] F_{\alpha}^{\prime 2}>-2 \Gamma \gamma(\beta) F_{\alpha}^{\prime} F_{\alpha}
$$

where

$$
\gamma(\beta) \equiv \frac{\beta+\mu(\beta)}{2 \beta+1}=\frac{\beta+\left[\left(\beta+\frac{1}{2}\right) /(2 \beta+1)\right]}{2 \beta+1}=\frac{1}{2}
$$

for all $\beta>0$; therefore, (28) is identical with

$$
F_{\alpha} F_{\alpha}^{\prime \prime}-[(\beta+1) /(2 \beta+1)] F_{\alpha}^{2} \geqslant-\Gamma F_{\alpha}^{\prime} F_{\alpha}
$$

for all $\beta>0$. Taking the limit in (30) as $\beta \rightarrow 0$ we obtain the desired differential inequality, i.e.,

$$
F_{\alpha} F_{\alpha}^{\prime \prime}-F_{\alpha}^{\prime 2} \geqslant-\Gamma F_{\alpha}^{\prime} F_{\alpha}
$$

Direct integration of (31) yields

$$
F_{\alpha}(t) \geqslant F_{\alpha}(0) \exp \left[\left\{F_{\alpha}^{\prime}(0) / \Gamma F_{\alpha}(0)\right\}\left(1-e^{-\Gamma t}\right)\right]
$$

and the desired estimate, i.e. (12), now follows directly from (5), with $u_{0}=\bar{u}_{0}$, 
and the definition of $F_{\alpha}$. If we now set

$$
\Sigma_{0}(\alpha, \Gamma)=\left\langle\bar{u}_{0}, v_{0}\right\rangle / \alpha \Gamma\left\|\bar{u}_{0}\right\|^{2},
$$

then it is immediate from (12) that

$$
\left\|u^{\alpha}(t)\right\|^{2} \geqslant \alpha^{2}\left\|\bar{u}_{0}\right\|^{2} e^{-f(t)},
$$

if $\alpha \geqslant\left\|v_{0}\right\| / \sqrt{\left\langle\bar{u}_{0}, N \bar{u}_{0}\right\rangle}$, where

$$
f(t) \equiv \Sigma_{0}(\alpha, \Gamma)\left(e^{-\Gamma t}-1\right)
$$

satisfies $f(0)=0$ and

$$
f^{\prime}(t)=-\Gamma \Sigma_{0}(\alpha, \Gamma) e^{-\Gamma t} \begin{cases}<0, & \left\langle\bar{u}_{0}, v_{0}\right\rangle_{H}>0, \\ >0, & \left\langle\bar{u}_{0}, v_{0}\right\rangle_{H}<0 .\end{cases}
$$

The asymptotic estimate claimed in (8) now follows directly from (33)-(35) if we let $t \rightarrow+\infty$ in (34) while the estimate delineated in (9) follows directly from (8) by taking the limit as $\Gamma \rightarrow \infty$ and using the definition (33) of $\Sigma_{0}(\alpha, \Gamma)$. Two other results are worth noting in passing, namely,

$$
\lim _{\alpha \rightarrow+\infty}\left(\left\|u^{\alpha}(t)\right\| / \alpha\right)^{2} \geqslant\left\|\bar{u}_{0}\right\|^{2}, \text { for all } t>0
$$

and, for $\alpha>\left\|v_{0}\right\| / \sqrt{\left\langle\bar{u}_{0}, N \bar{u}_{0}\right\rangle}$,

$$
\lim _{t \rightarrow+\infty}\left\|u^{\alpha}(t)\right\|^{2} \geqslant \alpha^{2}\left\|\bar{u}_{0}\right\|^{2} \exp \left\{-\left\|v_{0}\right\| / \alpha \Gamma\left\|\bar{u}_{0}\right\|\right\}
$$

which follows directly from (8), (33), and the Schwarz inequality.

As an application of the above results we may consider the equations of linear elasticity with viscous damping, i.e.,

$$
\ddot{u}_{i}^{\alpha}(x, t)+\Gamma \dot{u}_{i}^{\alpha}(x, t)-\frac{\partial}{\partial x_{j}}\left(c_{i j k l}(x) \frac{\partial u_{k}^{\alpha}(x, t)}{\partial x_{l}}\right)=0
$$

for $i=1,2,3, x \in \Omega \subseteq \Re^{3}$ (a bounded region with smooth boundary $\partial \Omega$ ) and $t>0 ; u$ is the elastic displacement vector while the $c_{i j k l}(x)$ are the components of the elasticities, $i, j, k, l=1,2,3$, which are assumed to satisfy the usual symmetries, i.e., $c_{i j k l}=c_{j i k l}=c_{k l i j}$. We take $H$ to be the completion of $C_{0}^{\infty}(\Omega)$ with respect to

$$
\langle\mathbf{u}, \mathbf{v}\rangle=\int_{\Omega} u_{i} v_{i} d x
$$

i.e., $H=L_{2}(\Omega)$ and define

$$
(N u)_{i}=\frac{\partial}{\partial x_{j}}\left(c_{i j k l}(x) \frac{\partial u_{k}}{\partial x_{l}}\right), \quad u \in H_{0}^{1}(\Omega),
$$

where $H_{0}^{1}(\Omega)$, the completion of $C_{0}^{\infty}(\Omega)$ with respect to

$$
\langle u, v\rangle_{+}=\int_{\Omega} \frac{\partial u_{i}}{\partial x_{j}} \frac{\partial v_{i}}{\partial x_{j}} d x
$$


satisfies $H_{0}^{1}(\Omega) \subseteq L_{2}(\Omega)$ algebraically and topologically with $H_{0}^{1}(\Omega)$ dense in $L_{2}(\Omega)$. We note that $N$, as defined by $(41)$ is bounded as a linear operator from $H_{0}^{1}(\Omega)$ into $H^{-1}(\Omega)$ where $H^{-1}(\Omega)$ is the completion of $C_{0}^{\infty}(\Omega)$ with respect to

$$
\|w\|_{-}=\sup _{v \in H_{0}^{1}(\Omega)}\left[\left|\int_{\Omega} w_{i} v_{i} d x\right| /\left(\int_{\Omega} \frac{\partial v_{i}}{\partial x_{j}} \frac{\partial v_{i}}{\partial x_{j}} d x\right)^{1 / 2}\right] .
$$

We append to (39) initial and boundary data of the form

$$
\begin{gathered}
u_{i}^{\alpha}(\mathbf{x}, t)=0, x \in \partial \Omega, t \geqslant 0, \\
u_{i}^{\alpha}(\mathbf{x}, 0)=\alpha f_{i}(x), \quad \dot{u}_{i}^{\alpha}(\mathbf{x}, 0)=g_{i}(x),
\end{gathered}
$$

where $f, g \in H_{0}^{1}(\Omega)$, and note that

$$
\langle f, N f\rangle=\int_{\Omega} f_{i} \frac{\partial}{\partial x_{j}}\left(c_{i j k l} \frac{\partial f_{k}}{\partial x_{l}}\right) d x=-\int_{\Omega} c_{i j k l} \frac{\partial f_{i}}{\partial x_{j}} \frac{\partial f_{k}}{\partial x_{l}} d x \geqslant 0
$$

provided the elasticities $c_{i j k l}(x)$ satisf $\mathrm{y}^{4}$

$$
\left.c_{i j k l}(x) \xi_{i j} \xi_{k l}<0 \text { (all } \xi \in \mathcal{L}\left(\Re^{3} ; \Re^{3}\right) \text { and } x \in \Omega\right) \text {. }
$$

If, in addition, there exists at least one element $\bar{f} \in H_{0}^{1}(\Omega)$ such that

$$
\int_{\Omega} c_{i j k l}(x) \frac{\partial \bar{f}_{i}}{\partial x_{j}} \frac{\partial \bar{f}_{k}}{\partial x_{l}} d x<0
$$

then the results which follow form the theorem above may be applied to the system consisting of (39), (44a) and (44b), with $f=\bar{f}$, provided we choose

$$
\alpha \geqslant\left(\int_{\Omega} g_{i} g_{i} d x\right)^{1 / 2} /\left[-\int_{\Omega} c_{i j k l} \frac{\partial \bar{f}_{i}}{\partial x_{j}} \frac{\partial \bar{f}_{k}}{\partial x_{l}} d x\right)^{1 / 2}
$$

\section{REFERENCES}

1. C. M. Dafermos, Wave equations with weak damping, SIAM J. Appl. Math. 18 (1970), 759-767.

2. D. L. Russell, Decay rates for weakly damped systems in Hilbert space obtained with control-theoretic methods, J. Differential Equations 19 (1975), 344-370.

3. H. A. Levine, Uniqueness and growth of weak solutions to certain linear differential equations in Hilbert space, J. Differential Equations 17 (1975), 73-81.

4. R. J. Knops and L. E. Payne, Growth estimates for solutions of evolutionary equations in Hilbert space with applications in elastodynamics, Arch. Rational Mech. Anal. 41 (1971), 363-398.

5. H. A. Levine, Logarithmic convexity, first order differential inequalities and some applications, Trans. Amer. Math. Soc. 152 (1970), 299-320.

6. Logarithmic convexity and the Cauchy problem for some abstract second order differential inequalities, J. Differential Equations 8 (1970), 34-55.

7. H. A. Levine and L. E. Payne, Nonexistence theorems for the heat equation with nonlinear boundary conditions and for the porous medium equation backward in time, J. Differential Equations 16 (1974), 319-334.

${ }^{4} \mathcal{L}\left(\Re^{3}, \Re^{3}\right)$ is the space of bounded linear operators from $\Re^{3}$ onto $\Re^{3}$. 
8. Some nonexistence theorems for initial-boundary value problems with nonlinear boundary constraints, Proc. Amer. Math. Soc. 46 (1974), 277-284.

9. H. A. Levine, Instability and nonexistence of global solutions to nonlinear wave equations of the form $\rho u=-A u+\mathcal{F}(u)$, Trans. Amer. Math. Soc. 192 (1974), 1-21.

10. Some nonexistence and instability theorems for formally parabolic equations of the form $\rho u_{t}=A u+\mathscr{F}(u)$, Arch. Rational Mech. Anal. 51 (1973), 371-386.

Departiment of Mathematics and Computer Science, University of South Carolina, Columbia, South Carolina 29208 